\title{
Characterizing mutual exclusivity as the strongest negative multivariate dependence structure
}

\author{
Ka Chun Cheung* and Ambrose Lo $^{\dagger}$ \\ Department of Statistics and Actuarial Science, The University of Hong Kong, \\ Pokfulam Road, Hong Kong
}

\begin{abstract}
Mutual exclusivity is an extreme negative dependence structure that was first proposed and studied in Dhaene and Denuit (1999) (The safest dependence structure among risks. Insurance: Mathematics and Economics 25, 11-21) in the context of insurance risks. In this article, we revisit this notion and present versatile characterizations of mutually exclusive random vectors via their pairwise counter-monotonic behaviour, minimal convex sum property, distributional representation and the characteristic function of the sum of their components. These characterizations highlight the role of mutual exclusivity in generalizing counter-monotonicity as the strongest negative dependence structure in a multi-dimensional setting.

Keywords: Mutual exclusivity; Fréchet bounds; Counter-monotonicity; Convex order; Complete mixability
\end{abstract}

\section{Introduction}

It is a fundamental result in the theory of dependence that the joint distribution function $F_{\mathbf{X}}$ of any random vector $\mathbf{X}$ is bounded from above and below by two multivariate functions:

$$
M_{n}\left(x_{1}, \ldots, x_{n}\right) \leq F_{\mathbf{X}}\left(x_{1}, \ldots, x_{n}\right) \leq W_{n}\left(x_{1}, \ldots, x_{n}\right) \text { for all }\left(x_{1}, \ldots, x_{n}\right) \in \mathbb{R}^{n} .
$$

Here, $W_{n}\left(x_{1}, \ldots, x_{n}\right)=\min \left\{F_{1}\left(x_{1}\right), \ldots, F_{n}\left(x_{n}\right)\right\}$ and $M_{n}\left(x_{1}, \ldots, x_{n}\right)=\left(\sum_{i=1}^{n} F_{i}\left(x_{i}\right)-n+1\right)_{+}$ are respectively known as the Fréchet upper bound and Fréchet lower bound of the Fréchet space $\mathcal{R}\left(F_{1}, \ldots, F_{n}\right)$ of all $n$-dimensional random vectors having $F_{1}, \ldots, F_{n}$ as marginal distributions. This classical result is known as the Fréchet-Höeffding inequality (see, for example, Joe (1997); Denuit et al. (2005)). While the Fréchet upper bound is always a proper distribution function

*Corresponding author. Email: kccg@hku.hk; Tel.: +852 28591987; fax: +852 28589041.

†Email: amblo@hku.hk; Tel.: +852 28597942. 
and corresponds to the extreme positive dependence structure of comonotonicity (see Dhaene et al. (2002) for further discussions), the Fréchet lower bound is in general not admissible in $\mathcal{R}\left(F_{1}, \ldots, F_{n}\right)$ when $n \geq 3$ (see Example 3.1 of Joe (1997)). This presumably explains why studies of multivariate negative dependence structure remain rare in the literature.

Necessary and sufficient conditions on the marginal distributions for $M_{n}, n \geq 3$, to be a proper distribution function are given in Joe (1997). When these conditions are fulfilled and the marginal distributions are restricted to be non-negative, the Fréchet lower bound corresponds to a structure known as mutual exclusivity studied in Dhaene and Denuit (1999). Among the many properties of a mutual exclusive random vector, the authors proved that this concept gives rise to the safest dependence structure among risks in that a mutually exclusive sum is the smallest with respect to convex order.

By giving manifold characterizations that parallel those of comonotonicity, this paper aims to justify rigorously the assertion that mutual exclusivity, whenever admissible, is an appropriate generalization of the bivariate notion of counter-monotonicity as the strongest negative multidimensional dependence structure. The organization of the paper is as follows. Section 2 is a recapitulation of the essential results needed in the sequel concerning the Fréchet lower bound, comonotonicity and counter-monotonicity. Known properties of mutual exclusivity considered in Dhaene and Denuit (1999) are reviewed in Section 3, where, to allow for greater generality, we also generalize the definition of mutually exclusive random variables by allowing their essential infima or essential suprema to be non-identical. Properties in the original definition are shown to carry over to the new one. The main results of this paper unfold in Section 4, where the first characterization of mutually exclusive (in the generalized sense defined in Section 3) random variables via their pairwise counter-monotonic behaviour is provided. The novel pairwise counter-monotonic property allows us to establish, in Section 5, that under appropriate conditions, a random vector is mutually exclusive if and only if the sum of its components is minimal in the sense of convex order. The relationship between mutual exclusivity and complete mixability, which is another negative dependence structure proposed in Wang and Wang (2011), is also delineated. Section 6 presents a new distributional representation of mutually exclusive random vectors, which sheds light on their structure and makes the computations of various expectations transparent. Section 7 is devoted to the analytic properties of mutual exclusivity. The necessary and sufficient conditions for the existence of mutually exclusive random vectors are articulated equivalently in terms of characteristic functions. In particular, it will be shown that, in contrast to comonotonicity, the characteristic function of mutually exclusive sums can be expressed explicitly in terms of the marginal characteristic functions. Two examples in risk theory enhance the interest and applicability of this result. Finally, Section 8 concludes the paper.

\section{Preliminaries}

All random variables in this paper are assumed to be defined on a common probability space $(\Omega, \mathcal{F}, \mathbb{P})$. The left-continuous inverse of the distribution function $F_{X}$ of any random variable $X$ is defined by

$$
F_{X}^{-1}(p):=\inf \left\{x \in \mathbb{R} \mid F_{X}(x) \geq p\right\}, \quad 0 \leq p \leq 1
$$


Similarly, the right-continuous inverse distribution function is defined by

$$
F_{X}^{-1+}(p):=\inf \left\{x \in \mathbb{R} \mid F_{X}(x)>p\right\}, \quad 0 \leq p \leq 1 .
$$

For any real $x$, we denote by $F_{X}\left(x^{-}\right)=\lim _{x_{n} \uparrow x} F_{X}\left(x_{n}\right)$ the left-hand limit of $F_{X}$ at $x$. The survival function and characteristic function of $X$ are denoted by $\bar{F}_{X}$ and $\varphi_{X}$ respectively. Integrability and non-degeneracy of all distributions are assumed.

\subsection{Fréchet lower bound}

The Fréchet lower bound $M_{n}$ is in general not a proper distribution function when $n \geq 3$. A necessary and sufficient condition for $M_{n}$ with $n \geq 3$ to be admissible in $\mathcal{R}\left(F_{1}, \ldots, F_{n}\right)$ is given in Theorem 3.7 of Joe (1997), which we state below for completeness.

Lemma 2.1. For $n \geq 3$, the Fréchet lower bound $M_{n}$ is a distribution function in $\mathcal{R}\left(F_{1}, \ldots, F_{n}\right)$ if and only if either of the following conditions holds:

(a) $\sum_{i=1}^{n} F_{i}\left(x_{i}\right) \leq 1$ for all $\left(x_{1}, \ldots, x_{n}\right) \in \mathbb{R}^{n}$ with $0<F_{i}\left(x_{i}\right)<1, i=1, \ldots$, n; or

(b) $\sum_{i=1}^{n} F_{i}\left(x_{i}\right) \geq n-1$ for all $\left(x_{1}, \ldots, x_{n}\right) \in \mathbb{R}^{n}$ with $0<F_{i}\left(x_{i}\right)<1, i=1, \ldots, n$.

The conditions in Lemma 2.1 can be interpreted as follows. Condition (a) means that for each $F_{i}$, there is a finite upper support point $u_{i}$ on which $F_{i}$ has a probability mass, and the probability masses $1-F_{i}\left(u_{i}^{-}\right)$at the $u_{i}$ 's are large enough in the sense that $\sum_{i=1}^{n}\left[1-F_{i}\left(u_{i}^{-}\right)\right] \geq n-1$. Condition (b) admits a similar interpretation.

\subsection{Comonotonicity}

Comonotonicity as the strongest positive dependence structure has been studied extensively in the literature (see Dhaene et al. (2002) and Deelstra et al. (2011) for overviews of the most relevant results of this dependency notion). A random vector $\mathbf{X}=\left(X_{1}, \ldots, X_{n}\right)$ is said to be comonotonic if there is a null set $N$ such that $\left(X_{i}(\omega)-X_{i}\left(\omega^{\prime}\right)\right)\left(X_{j}(\omega)-X_{j}\left(\omega^{\prime}\right)\right) \geq 0$ for all $i, j \in\{1, \ldots, n\}$ and $\omega, \omega^{\prime} \in \Omega \backslash N$.

The following lemma collects several characterizations of comonotonicity concerning the joint distribution, pairwise comonotonic property, maximal convex sum property and distributional representation of a comonotonic random vector. They can be found, for example, in Dhaene et al. (2002); Cheung (2008, 2010). Two swift proofs of Property (c) are discussed in Remark 4 of Cheung and Lo (2013a).

Lemma 2.2. A random vector $\mathbf{X}^{*}=\left(X_{1}^{*}, \ldots, X_{n}^{*}\right)$ in the Fréchet space $\mathcal{R}\left(F_{1}, \ldots, F_{n}\right)$ is comonotonic if and only if the following equivalent conditions hold:

(a) $F_{\mathbf{X}^{*}}(\mathbf{x})=W_{n}(\mathbf{x})$ for all $\mathbf{x}=\left(x_{1}, \ldots, x_{n}\right) \in \mathbb{R}^{n}$; 
(b) $\left(X_{i}^{*}, X_{j}^{*}\right)$ is comonotonic for all $i \neq j$;

(c) $X_{1}+\cdots+X_{n} \leq_{c x} X_{1}^{*}+\cdots+X_{n}^{*}$ for all $\left(X_{1}, \ldots, X_{n}\right) \in \mathcal{R}\left(F_{1}, \ldots, F_{n}\right)$;

(d) for any uniform $(0,1)$ random variable $U$,

$$
\mathbf{X} \stackrel{d}{=}\left(F_{1}^{-1}(U), \ldots, F_{n}^{-1}(U)\right)
$$

The objective of this paper is to show that each of these properties enjoys a parallel version for mutually exclusive random vectors, suggesting that mutual exclusivity is the structure giving rise to the most negative dependence.

\subsection{Counter-monotonicity}

In the special case of $n=2$, the Fréchet lower bound $M_{2}$ corresponds to an extreme dependence structure known as counter-monotonicity. A bivariate random vector $\left(X_{1}, X_{2}\right)$ is said to be counter-monotonic if there is a null set $N$ such that $\left(X_{1}(\omega)-X_{1}\left(\omega^{\prime}\right)\right)\left(X_{2}(\omega)-X_{2}\left(\omega^{\prime}\right)\right) \leq 0$ for any $\omega, \omega^{\prime} \in \Omega \backslash N$. Since $X_{1}$ and $X_{2}$ move in the opposite direction almost surely, this explains why $\left(X_{1}, X_{2}\right)$ exhibits the strongest negative dependence. Note that counter-monotonicity is a bivariate concept. An application of counter-monotonicity to merging risks was considered in Cheung et al. (2014).

The key step in the proofs of our main results lies in reducing multivariate problems to bivariate ones and applying known results of counter-monotonicity. To this end, the following result, which asserts that the minimal convex sum behaviour of a random vector is a defining property of counter-monotonicity, will play an instrumental role in connecting counter-monotonicity and mutual exclusivity. The "only if" part is a standard result (see, for example, Dhaene and Goovaerts (1996); Denuit et al. (2005)), while the "if" part was proved recently in Theorem 1 and Remark 1 of Cheung and Lo (2013a) using three simple methods.

Lemma 2.3. A bivariate random vector $\left(X_{1}^{*}, X_{2}^{*}\right)$ in $\mathcal{R}\left(F_{1}, F_{2}\right)$ is counter-monotonic if and only if

$$
X_{1}^{*}+X_{2}^{*} \leq_{c x} X_{1}+X_{2} \quad \text { for all }\left(X_{1}, X_{2}\right) \in \mathcal{R}\left(F_{1}, F_{2}\right)
$$

A generalization of Lemma 2.3 to three or more random variables will be demonstrated in Section 5 of this paper.

\section{Generalized definition of mutually exclusive random variables}

The notion of mutually exclusive random variables was first proposed by Dhaene and Denuit (1999) in the context of non-negative random variables, more commonly known as risks. 
Definition 3.1. (Dhaene and Denuit (1999), Definition 6) The non-negative random variables $X_{1}, \ldots, X_{n}$ are said to be mutually exclusive if

$$
\mathbb{P}\left(X_{i}>0, X_{j}>0\right)=0 \text { for all } i \neq j .
$$

As noted in Dhaene and Denuit (1999), examples of mutually exclusive random variables abound in actuarial science and finance. For instance, the present value random variables of term and endowment insurances written on the same life are mutually exclusive, so are the payoffs of European call and put options written on the same underlying asset with the same strike price and time to expiration.

As the notion of mutual exclusivity imposes probability masses on zero, an arbitrary Fréchet space of non-negative distributions, such as continuous distributions, does not always contain mutually exclusive risks. A necessary and sufficient condition for mutual exclusivity to be a feasible dependence structure was given in Theorem 7 of Dhaene and Denuit (1999).

Lemma 3.2. (Dhaene and Denuit (1999), Theorem 7) A Fréchet space $\mathcal{R}\left(F_{1}, \ldots, F_{n}\right)$ of nonnegative distributions supports mutually exclusive random variables if and only if the condition $\sum_{i=1}^{n}\left[1-F_{i}(0)\right] \leq 1$ is fulfilled.

Some useful properties of mutually exclusive random variables derived in Dhaene and Denuit (1999) are collected in Lemma 3.3. Property (a) shows that the joint distribution of a mutually exclusive random vector reaches precisely the Fréchet lower bound, and Property (b) demonstrates the additivity of the stop-loss premiums of a sum of mutually exclusive random variables for non-negative deductibles. This implies the substantive fact in (c) that a mutually exclusive sum, when it exists, is minimal with respect to convex order. For two random variables $X$ and $Y$, we say that $X$ is less than $Y$ in convex order if $\mathbb{E}[X]=\mathbb{E}[Y]$ and $\mathbb{E}\left[(X-t)_{+}\right] \leq \mathbb{E}\left[(Y-t)_{+}\right]$ for all real $t$ (see Shaked and Shanthikumar (2007)).

Lemma 3.3. Let $\mathbf{X}^{*}=\left(X_{1}^{*}, \ldots, X_{n}^{*}\right)$ be a random vector in a Fréchet space $\mathcal{R}\left(F_{1}, \ldots, F_{n}\right)$ of non-negative distributions satisfying $\sum_{i=1}^{n}\left[1-F_{i}(0)\right] \leq 1$, and $S^{*}=X_{1}^{*}+\cdots+X_{n}^{*}$.

(a) $\mathbf{X}^{*}$ is mutually exclusive if and only if $F_{\mathbf{X}^{*}}=M_{n}$;

if $\mathbf{X}^{*}$ is mutually exclusive, then:

(b) $\mathbb{E}\left[\left(S^{*}-d\right)_{+}\right]=\sum_{i=1}^{n} \mathbb{E}\left[\left(X_{i}^{*}-d\right)_{+}\right]$for all $d \geq 0$;

(c) $X_{1}^{*}+\cdots+X_{n}^{*} \leq_{c x} X_{1}+\cdots+X_{n}$ for all $\left(X_{1}, \ldots, X_{n}\right) \in \mathcal{R}\left(F_{1}, \ldots, F_{n}\right)$.

Motivated by the two conditions in Lemma 2.1, in this section we propose a more generalized definition of mutually exclusive random variables to be used hereafter to allow for considerably greater generality. Further explanations on why the generalized definition is desirable will be given in Remark $4.2(\mathrm{~d})$ in the next section. Throughout this paper, we let $l_{i}$ and $u_{i}$ be the essential infimum and essential supremum of a random variable $X_{i}$, i.e. $l_{i}=F_{X_{i}}^{-1+}(0)$ and $u_{i}=F_{X_{i}}^{-1}(1)$. 
Definition 3.4. (Mutual exclusivity in the generalized sense) The real-valued random variables $X_{1}, \ldots, X_{n}$ are said to be:

(a) mutually exclusive from below if $\mathbb{P}\left(X_{i}>l_{i}, X_{j}>l_{j}\right)=0$ for all $i \neq j$;

(b) mutually exclusive from above if $\mathbb{P}\left(X_{i}<u_{i}, X_{j}<u_{j}\right)=0$ for all $i \neq j$.

In other words, if $X_{1}, \ldots, X_{n}$ are mutually exclusive, then the realized value of at most one of them can differ from its essential infimum or essential supremum. Intuitively, such restricted pointwise behavior exerts exceedingly strong negative dependence on the underlying random variables. Note that implicit in the definition is the fact that $l_{1}, \ldots, l_{n}$ or $u_{1}, \ldots, u_{n}$ are all real-valued; otherwise, $X_{1}, \ldots, X_{n}$ would be degenerated at $+\infty$ or $-\infty$.

By translating the random variables back to the original definition of mutual exclusivity, direct analogues of Lemmas 3.2 and 3.3 can be readily obtained.

Lemma 3.5. For $n \geq 3$, a Fréchet space $\mathcal{R}\left(F_{1}, \ldots, F_{n}\right)$ accommodates mutually exclusive random variables if and only if any of the following conditions is satisfied:

(a) $\sum_{i=1}^{n} q_{i} \leq 1$, where $q_{i}:=1-F_{i}\left(l_{i}\right), i=1, \ldots, n$, in which case random variables which are mutually exclusive from below are supported;

(b) $\sum_{i=1}^{n} p_{i} \leq 1$, where $p_{i}:=F_{i}\left(u_{i}^{-}\right), i=1, \ldots, n$, in which case random variables that are mutually exclusive from above are supported.

From the remark following Lemma 2.1, we note that Condition (a) (resp. (b)) in Lemma 3.5 is equivalent to Condition (b) (resp. (a)) in Lemma 2.1. In other words, the two conditions in Lemma 3.5 are also necessary and sufficient conditions for the Fréchet lower bound $M_{n}$ to be a genuine distribution function when $n \geq 3$.

Throughout this paper, we denote the two mutually exclusive conditions in Lemma 3.5 by Condition (A):

$$
\sum_{i=1}^{n} q_{i} \leq 1, \text { where } q_{i}:=1-F_{i}\left(l_{i}\right), i=1, \ldots, n,
$$

Condition (A): or

$$
\sum_{i=1}^{n} p_{i} \leq 1, \text { where } p_{i}:=F_{i}\left(u_{i}^{-}\right), i=1, \ldots, n \text {. }
$$

We will also emphasize instances in which Condition (A) is a hypothesis and those in which it is a consequence. Notation-wise, we denote by $\mathbf{X}^{M B}$ the counterpart of a given random vector $\mathbf{X}$ which is mutually exclusive from below, and by $\mathbf{X}^{M A}$ the random vector that is mutually exclusive from above. When both possibilities wish to be incorporated, the generic term "mutually exclusive" will be used without "from below" or "from above", and a mutually exclusive random vector will be written as $\mathbf{X}^{M}$. The sum of the components of $\mathbf{X}^{M B}, \mathbf{X}^{M A}$ and $\mathbf{X}^{M}$ are denoted by $S^{M B}, S^{M A}$ and $S^{M}$ respectively. 
Lemma 3.6. Let $\mathbf{X}^{*}$ be a random vector in a Fréchet space $\mathcal{R}\left(F_{1}, \ldots, F_{n}\right)$ with $n \geq 3$.

(a) $\mathbf{X}^{*}$ is mutually exclusive if and only if $F_{\mathbf{X}^{*}}=M_{n}$;

(b) if $\mathbf{X}^{*}$ is mutually exclusive, then

$$
X_{1}^{*}+\cdots+X_{n}^{*} \leq_{c x} X_{1}+\cdots+X_{n} \quad \text { for all }\left(X_{1}, \ldots, X_{n}\right) \in \mathcal{R}\left(F_{1}, \ldots, F_{n}\right) .
$$

Proof. Since the proof of (a) is highly similar to that of Theorem 8 of Dhaene and Denuit (1999), we only prove (b).

Case 1. Suppose that $\mathbf{X}^{*}$ is mutually exclusive from below such that the condition $\sum_{i=1}^{n} q_{i} \leq$ 1 in Lemma 3.5 (a) holds. For all $d \geq \sum_{i=1}^{n} l_{i}$ and $\left(X_{1}, \ldots, X_{n}\right) \in \mathcal{R}\left(F_{1}, \ldots, F_{n}\right)$, we apply Lemma 3.3 (b) to the non-negative mutually exclusive random variables $X_{i}-l_{i}$ and obtain

$$
\begin{aligned}
\mathbb{E}\left[\left(\sum_{i=1}^{n} X_{i}^{*}-d\right)_{+}\right] & =\mathbb{E}\left[\sum_{i=1}^{n}\left(X_{i}^{*}-l_{i}\right)-\left(d-\sum_{i=1}^{n} l_{i}\right)\right]_{+} \\
& =\sum_{i=1}^{n} \mathbb{E}\left[\left(X_{i}-l_{i}\right)-\left(d-\sum_{i=1}^{n} l_{i}\right)\right]_{+} .
\end{aligned}
$$

Due to the superadditivity of the function $f(x)=(x-d)_{+}$for any $x \geq 0$ and $d \geq 0$, we further have

$$
\begin{aligned}
\sum_{i=1}^{n} \mathbb{E}\left[\left(X_{i}-l_{i}\right)-\left(d-\sum_{i=1}^{n} l_{i}\right)\right]_{+} & \leq \mathbb{E}\left[\sum_{i=1}^{n}\left(X_{i}-l_{i}\right)-\left(d-\sum_{i=1}^{n} l_{i}\right)\right]_{+} \\
& =\mathbb{E}\left[\left(\sum_{i=1}^{n} X_{i}-d\right)_{+}\right] .
\end{aligned}
$$

Combining (1) and (2), we get

$$
\mathbb{E}\left[\left(\sum_{i=1}^{n} X_{i}^{*}-d\right)_{+}\right] \leq \mathbb{E}\left[\left(\sum_{i=1}^{n} X_{i}-d\right)_{+}\right]
$$

for any $d \geq \sum_{i=1}^{n} l_{i}$. Since (3) is trivially true for $d<\sum_{i=1}^{n} l_{i}$, we conclude that $X_{1}^{*}+\cdots+X_{n}^{*} \leq_{c x} X_{1}+\cdots+X_{n}$.

Case 2. If $\mathbf{X}^{*}$ is mutually exclusive from above, then $-\mathbf{X}^{*}$ is mutually exclusive from below. Applying the result in Case 1, one finds

$$
-\sum_{i=1}^{n} X_{i}^{*} \leq_{c x}-\sum_{i=1}^{n} X_{i}
$$

or equivalently,

$$
\sum_{i=1}^{n} X_{i}^{*} \leq_{c x} \sum_{i=1}^{n} X_{i}
$$

for all $\left(X_{1}, \ldots, X_{n}\right) \in \mathcal{R}\left(F_{1}, \ldots, F_{n}\right)$. 
Remark 3.7. (a) It should be noted that unlike Lemma 3.3, Lemma 3.6 (a) is in general not true when $n=2$. In the bivariate case, $M_{2}$ is always a distribution function, so one cannot conclude from $F_{\mathbf{X}}=M_{2}$ that $\mathbf{X}$ must be mutually exclusive. For a simple counter-example, consider $\left(X_{1}, X_{2}\right) \stackrel{d}{=}(U, 1-U)$ for any uniform $(0,1)$ random variable $U$.

(b) In fact, Lemma 3.6 (b) can be proved more easily by applying Lemma 3.3 (c) to the non-negative mutually exclusive random variables $X_{i}^{*}-l_{i}$, yielding

$$
\sum_{i=1}^{n} X_{i}^{*}-\sum_{i=1}^{n} l_{i} \leq_{c x} \sum_{i=1}^{n} X_{i}-\sum_{i=1}^{n} l_{i}
$$

for all $\left(X_{1}, \ldots, X_{n}\right) \in \mathcal{R}\left(F_{1}, \ldots, F_{n}\right)$. The result follows by eliminating the constant $\sum_{i=1}^{n} l_{i}$ on both sides. Nevertheless, (1) and (2) shall be of use in later sections.

\section{Pairwise counter-monotonic property of a mutually exclusive random vector}

It is well-known that a random vector with three or more random variables is comonotonic if and only if it is pairwise comonotonic (Lemma 2.2 (b)). When pairwise comonotonicity is replaced by pairwise counter-monotonicity, the resulting dependence structure, even if it exists, is still unknown in the literature due to the eccentric pointwise behavior of the whole random vector. For such a random vector, when one component increases and another decreases, all remaining components must remain stationary almost surely, resulting in some probability masses on the marginal distributions required in the definition of mutual exclusivity. The goal of this section is to show that the pairwise counter-monotonicity property is synonymous with mutual exclusivity, either from above or below. This result is interesting in its own right and will be used in the next section to characterize mutual exclusivity further.

Theorem 4.1. Let $\mathbf{X}=\left(X_{1}, \ldots, X_{n}\right)$ be a random vector in $\mathcal{R}\left(F_{1}, \ldots, F_{n}\right)$ with $n \geq 3$. Then $\mathbf{X}$ is mutually exclusive if and only if $\left(X_{i}, X_{j}\right)$ is counter-monotonic for all $i \neq j$.

Proof. Assume that $\mathbf{X}$ is mutually exclusive. By Lemma 3.6 (a), the distribution function of $\mathbf{X}$ is $M_{n}$, so that of $\left(X_{i}, X_{j}\right)$ for any $i \neq j$ is

$$
\begin{aligned}
F_{X_{i}, X_{j}}\left(x_{i}, x_{j}\right) & =\lim _{\substack{k \rightarrow \infty \\
k \neq i, j}} M_{n}\left(x_{1}, \ldots, x_{i}, \ldots, x_{j}, \ldots, x_{n}\right) \\
& =\left[F_{i}\left(x_{i}\right)+F_{j}\left(x_{j}\right)+(n-2)-n+1\right]_{+} \\
& =M_{2}\left(x_{i}, x_{j}\right)
\end{aligned}
$$

for all $\left(x_{i}, x_{j}\right) \in \mathbb{R}^{2}$. Reaching the Fréchet lower bound, $\left(X_{i}, X_{j}\right)$ is counter-monotonic.

Conversely, suppose that $\left(X_{i}, X_{j}\right)$ is counter-monotonic whenever $i \neq j$. Without loss of generality, we assume $n=3$ and consider three pairwise counter-monotonic random variables, $X_{1}, X_{2}$ 
and $X_{3}$. It is then enough to show that the distribution function of $\mathbf{X}=\left(X_{1}, X_{2}, X_{3}\right)$ is given by

$$
F_{\mathbf{X}}(\mathbf{x})=M_{3}(\mathbf{x})=\left(\sum_{i=1}^{3} F_{i}\left(x_{i}\right)-2\right)_{+}, \quad \text { for all } \mathbf{x} \in \mathbb{R}^{3} .
$$

This is because if this is true, then $M_{3}$ is a genuine distribution function in $\mathcal{R}\left(F_{1}, F_{2}, F_{3}\right)$. By Lemmas 2.1 and 3.5, Condition (A) holds and $\mathcal{R}\left(F_{1}, F_{2}, F_{3}\right)$ supports a mutually exclusive random vector $\mathbf{X}^{M}=\left(X_{1}^{M}, X_{2}^{M}, X_{3}^{M}\right)$ whose distribution function, by virtue of Lemma 3.6 (a), is $M_{3}$. Hence $\mathbf{X}$ and $\mathbf{X}^{M}$ coincide in distribution, which means that $\mathbf{X}$ is also a mutually exclusive random vector.

For any $\mathbf{x} \in \mathbb{R}^{3}$ such that $F_{\mathbf{X}}(\mathbf{x})=0$, the Fréchet-Höeffding inequality implies that

$$
0 \leq M_{3}(\mathbf{x}) \leq F_{\mathbf{X}}(\mathbf{x})=0
$$

Thus $F_{\mathbf{X}}(\mathbf{x})=M_{3}(\mathbf{x})=0$. To show (4) for $\mathbf{x} \in \mathbb{R}^{3}$ such that $F_{\mathbf{X}}(\mathbf{x})>0$, we divide the remainder of the proof into several steps.

Step 1: Fix $\mathbf{x} \in \mathbb{R}^{3}$ such that $\mathbb{P}(\mathbf{X} \in L)=F_{\mathbf{X}}(\mathbf{x})>0$, where

$$
L:=\left\{\mathbf{y} \in \mathbb{R}^{3} \mid y_{i} \leq x_{i} \text { for all } i=1,2,3\right\} .
$$

For any distinct $i$ and $j$ in $\{1,2,3\}$, let

$$
L_{i j}:=\left\{\mathbf{y} \in \mathbb{R}^{3} \mid y_{i} \leq x_{i}, y_{j} \leq x_{j}\right\}
$$

Then we have

$$
L=\bigcap_{i \neq j} L_{i j}=L_{12} \cap L_{23}=L_{12} \cap L_{13}=L_{13} \cap L_{23}=L_{12} \cap L_{13} \cap L_{23} .
$$

Step 2: We claim that

$$
\mathbb{P}\left(\mathbf{X} \in \bigcup_{i \neq j} L_{i j}\right)=1
$$

To show this, we exploit the pairwise counter-monotonic hypothesis and let $N$ be a null set such that whenever $\omega, \omega^{\prime} \in \Omega \backslash N$,

$$
\left(X_{i}(\omega)-X_{i}\left(\omega^{\prime}\right)\right)\left(X_{j}(\omega)-X_{j}\left(\omega^{\prime}\right)\right) \leq 0 \quad \text { for all } i \neq j .
$$

Then $S:=\{\mathbf{X}(\omega) \mid \omega \in \Omega \backslash N\}$ is a support of $\mathbf{X}$. Since $\mathbb{P}(\mathbf{X} \in L)>0, L \cap S$ is non-empty, so there exists $\mathbf{a} \in L \cap S$, which means

$$
a_{i} \leq x_{i} \text { for all } i=1,2,3 \text {. }
$$

If $\left(\bigcap_{i \neq j} L_{i j}^{C}\right) \cap S$ is non-empty, then we can find $\mathbf{b} \in\left(\bigcap_{i \neq j} L_{i j}^{C}\right) \cap S$ such that there exist distinct $i^{*}$ and $j^{*}$ in $\{1,2,3\}$ such that

$$
b_{i^{*}}>x_{i^{*}} \quad \text { and } \quad b_{j^{*}}>x_{j^{*}} .
$$


Combining (7) and (8), we obtain

$$
\left(a_{i^{*}}-b_{i^{*}}\right)\left(a_{j^{*}}-b_{j^{*}}\right)>0,
$$

which contradicts the fact that $\mathbf{a}, \mathbf{b} \in S$. In other words, $\left(\bigcap_{i \neq j} L_{i j}^{C}\right) \cap S$ must be empty, so (6) holds.

Step 3: From (6),

$$
\begin{aligned}
1= & \mathbb{P}\left(\mathbf{X} \in L_{12} \cup L_{23} \cup L_{13}\right) \\
= & \mathbb{P}\left(\mathbf{X} \in L_{12}\right)+\mathbb{P}\left(\mathbf{X} \in L_{23}\right)+\mathbb{P}\left(\mathbf{X} \in L_{13}\right) \\
& \quad-\mathbb{P}\left(\mathbf{X} \in L_{12} \cap L_{23}\right)-\mathbb{P}\left(\mathbf{X} \in L_{12} \cap L_{13}\right) \\
& \quad-\mathbb{P}\left(\mathbf{X} \in L_{23} \cap L_{13}\right)+\mathbb{P}\left(\mathbf{X} \in L_{12} \cap L_{23} \cap L_{13}\right) \\
= & \mathbb{P}\left(\mathbf{X} \in L_{12}\right)+\mathbb{P}\left(\mathbf{X} \in L_{23}\right)+\mathbb{P}\left(\mathbf{X} \in L_{13}\right)-2 \mathbb{P}(\mathbf{X} \in L) \\
= & \left(F_{1}\left(x_{1}\right)+F_{2}\left(x_{2}\right)-1\right)_{+}+\left(F_{2}\left(x_{2}\right)+F_{3}\left(x_{3}\right)-1\right)_{+} \\
& \quad+\left(F_{1}\left(x_{1}\right)+F_{3}\left(x_{3}\right)-1\right)_{+}-2 F_{\mathbf{X}}(\mathbf{x}),
\end{aligned}
$$

where the third equality follows from (5), and the last equality is due to the pairwise countermonotonicity of $\mathbf{X}$. For notational simplicity, we let $t_{i}:=F_{i}\left(x_{i}\right)$ for $i=1,2,3$, and

$$
\begin{aligned}
& T_{1}:=\left(t_{2}+t_{3}-1\right)_{+}, \\
& T_{2}:=\left(t_{1}+t_{3}-1\right)_{+}, \\
& T_{3}:=\left(t_{1}+t_{2}-1\right)_{+} .
\end{aligned}
$$

Then we have

$$
F_{\mathbf{X}}(\mathbf{x})=\frac{1}{2}\left(T_{1}+T_{2}+T_{3}-1\right)
$$

Note that $F_{\mathbf{X}}(\mathbf{x})>0$ by our choice of $\mathbf{x}$, and each $T_{i} \leq 1$, so among $T_{1}, T_{2}, T_{3}$, at least two of them must be strictly positive.

- Case 1 ( $T_{1}, T_{2}, T_{3}$ are all strictly positive): In this case,

$$
\begin{aligned}
F_{\mathbf{X}}(\mathbf{x}) & =\frac{1}{2}\left[2\left(t_{1}+t_{2}+t_{3}\right)-4\right] \\
& =t_{1}+t_{2}+t_{3}-2 \\
& =M_{3}(\mathbf{x}) .
\end{aligned}
$$

- Case 2 (one $T_{i}$ is zero): Without loss of generality, suppose that $T_{1}=0$ and $T_{2}>0, T_{3}>$ 0 . Then

$$
\begin{aligned}
F_{\mathbf{X}}(\mathbf{x}) & =\frac{1}{2}\left[\left(t_{1}+t_{3}-1\right)+\left(t_{1}+t_{2}-1\right)-1\right] \\
& =\frac{1}{2}\left(2 t_{1}+t_{2}+t_{3}-3\right) .
\end{aligned}
$$

However, $T_{1}=0$ implies that $t_{2}+t_{3} \leq 1$, which in turn leads to

$$
F_{\mathbf{X}}(\mathbf{x}) \leq \frac{1}{2}[2(1)+(1)-3]=0
$$

which is a contradiction. 
Therefore, Case 1 is the only possibility. This completes the proof of (4) and, as a result, that of Theorem 4.1 .

Remark 4.2. (a) Theorem 4.1 not only reveals the intimate relationship between mutual exclusivity and counter-monotonicity, but also suggests that the study of mutual exclusivity boils down to that of bivariate distributions. This is a characteristic of an extreme dependence structure such as comonotonicity. This approach will be used in the proof of Theorem 5.1.

(b) The same argument in Remark 3.7 (a) shows that Theorem 4.1 is generally not true when $n=2$.

(c) Note that Condition (A) is a direct consequence of Theorem 4.1 and Lemma 3.5; it need not be imposed for Theorem 4.1 to hold true. If it is known a priori that the underlying Fréchet space satisfies Condition (A) (as assumed throughout in Dhaene and Denuit (1999)), the proof of the sufficiency part of Theorem 4.1 can be considerably simplified as follows:

Suppose that $\sum_{i=1}^{n} q_{i} \leq 1$ and fix any distinct indices $j$ and $k$. Since $q_{i} \geq 0$ for all $i=1, \ldots, n$, the condition $\sum_{i=1}^{n} q_{i} \leq 1$ implies that $q_{j}+q_{k} \leq 1$. By Lemma 3.5 (a), the two-dimensional Fréchet space $\mathcal{R}\left(F_{j}, F_{k}\right)$ supports mutually exclusive (from below) random variables. Then it follows that $\left(X_{j}, X_{k}\right)$, with distribution $M_{2}$ and lying in $\mathcal{R}\left(F_{j}, F_{k}\right)$, is not only counter-monotonic, but also mutually exclusive from below for all $j \neq k$. If $X_{i}>l_{i}$ for some $i \in\{1, \ldots, n\}$, then by the definition of mutual exclusivity, $X_{j}=l_{j}$ for all $j \neq i$, which shows that the whole random vector $\mathbf{X}$ is mutually exclusive from below.

The case of $\sum_{i=1}^{n} p_{i} \leq 1$ is similar.

The advantage of the proof of the sufficiency part of Theorem 4.1 is that we directly show that the random vector must be mutually exclusive without making use of Condition (A), which is a by-product of the theorem, because the pairwise counter-monotonicity property itself imposes strong restrictions on the Fréchet space.

(d) With the pairwise counter-monotonic property alone, we can neither determine the signs of the $l_{i}$ 's and $u_{i}$ 's nor conclude whether $\mathbf{X}$ is mutually exclusive from below or from above. This explains the necessity of introducing the generalized definition of mutual exclusivity in Section 3.

(e) The non-degeneracy assumption, which is made throughout this paper, is crucial as illustrated by the random vector $(U, 1,1-U)$, where $U$ is any uniform $(0,1)$ random variable. This random vector is pairwise counter-monotonic, but not mutually exclusive from above or below.

\section{Minimal convex sum property}

As a further step towards fully characterizing mutual exclusivity, in this section we show that mutually exclusive random variables are defined by the minimality of their sum with respect 
to convex order. The main idea of our proof is to reduce multivariate assumptions to bivariate statements, apply the minimal convex sum property of counter-monotonicity applicable to bivariate couples and use the pairwise counter-monotonic property established in the previous section to arrive at the desired multivariate conclusion.

Theorem 5.1. Let $\mathbf{X}^{*}=\left(X_{1}^{*}, \ldots, X_{n}^{*}\right)$ be a fixed random vector in the Fréchet space $\mathcal{R}\left(F_{1}, \ldots, F_{n}\right)$ which satisfies Condition (A). Then $\mathbf{X}^{*}$ is mutually exclusive if and only if

$$
X_{1}^{*}+\cdots+X_{n}^{*} \leq_{c x} X_{1}+\cdots+X_{n} \quad \text { for all }\left(X_{1}, \ldots, X_{n}\right) \in \mathcal{R}\left(F_{1}, \ldots, F_{n}\right) .
$$

Proof. The necessity follows from Lemma 3.6 (b). For the converse, we take a mutually exclusive random vector $\mathbf{X}^{M}$ in $\mathcal{R}\left(F_{1}, \ldots, F_{n}\right)$, which exists under Condition (A), and assume without loss of generality that $\mathbf{X}^{M}$ is mutually exclusive from below. Then Lemma 3.6 (b) and the hypothesis together imply

$$
X_{1}^{*}+\cdots+X_{n}^{*} \stackrel{d}{=} X_{1}^{M}+\cdots+X_{n}^{M}
$$

We now prove that

$$
X_{1}^{*}+\cdots+X_{n-1}^{*} \stackrel{d}{=} X_{1}^{M}+\cdots+X_{n-1}^{M}
$$

by showing that the stop-loss premiums on both sides are equal. To this end, we proceed as in the proof of Lemma 3.6 (b) and write the stop-loss premium of the mutually exclusive sum in (9) as

$$
\begin{aligned}
\mathbb{E}\left[\left(\sum_{i=1}^{n} X_{i}^{M}-d\right)_{+}\right] & =\sum_{i=1}^{n-1} \mathbb{E}\left[\left(X_{i}^{M}-l_{i}\right)-\left(d-\sum_{i=1}^{n} l_{i}\right)\right]_{+}+\mathbb{E}\left[\left(X_{n}^{M}-l_{n}\right)-\left(d-\sum_{i=1}^{n} l_{i}\right)\right]_{+} \\
& =\mathbb{E}\left[\sum_{i=1}^{n-1}\left(X_{i}^{M}-l_{i}\right)-\left(d-\sum_{i=1}^{n} l_{i}\right)\right]_{+}+\mathbb{E}\left[\left(X_{n}^{M}-l_{n}\right)-\left(d-\sum_{i=1}^{n} l_{i}\right)\right]_{+} \\
& =\mathbb{E}\left[\sum_{i=1}^{n-1} X_{i}^{M}-\left(d-l_{n}\right)\right]_{+}+\mathbb{E}\left[X_{n}^{M}-\left(d-\sum_{i=1}^{n-1} l_{i}\right)\right]_{+},
\end{aligned}
$$

for all $d \geq \sum_{i=1}^{n} l_{i}$, where the second equality follows because $\left(X_{1}^{M}, \ldots, X_{n-1}^{M}\right)$ is also mutually exclusive. On the other hand, we also have

$$
\begin{aligned}
\mathbb{E}\left[\left(\sum_{i=1}^{n} X_{i}^{*}-d\right)_{+}\right] & =\mathbb{E}\left[\sum_{i=1}^{n}\left(X_{i}^{*}-l_{i}\right)-\left(d-\sum_{i=1}^{n} l_{i}\right)\right]_{+} \\
& \geq \mathbb{E}\left[\sum_{i=1}^{n-1}\left(X_{i}^{*}-l_{i}\right)-\left(d-\sum_{i=1}^{n} l_{i}\right)\right]_{+}+\mathbb{E}\left[\left(X_{n}^{*}-l_{n}\right)-\left(d-\sum_{i=1}^{n} l_{i}\right)\right]_{+} \\
& =\mathbb{E}\left[\sum_{i=1}^{n-1} X_{i}^{*}-\left(d-l_{n}\right)\right]_{+}+\mathbb{E}\left[X_{n}^{*}-\left(d-\sum_{i=1}^{n-1} l_{i}\right)\right]_{+}
\end{aligned}
$$

for all $d \geq \sum_{i=1}^{n} l_{i}$. Because of (9), we combine (11) and (12) to obtain

$$
\mathbb{E}\left[\sum_{i=1}^{n-1} X_{i}^{M}-\left(d-l_{n}\right)\right]_{+} \geq \mathbb{E}\left[\sum_{i=1}^{n-1} X_{i}^{*}-\left(d-l_{n}\right)\right]_{+}
$$


for all $d \geq \sum_{i=1}^{n} l_{i}$. Note that (13) is trivially true for $d<\sum_{i=1}^{n} l_{i}$, so (13) indeed holds for all $d \in \mathbb{R}$. Since the reverse inequality must also hold by Lemma 3.6 (b), we have

$$
\mathbb{E}\left[\left(\sum_{i=1}^{n-1} X_{i}^{M}-d\right)_{+}\right]=\mathbb{E}\left[\left(\sum_{i=1}^{n-1} X_{i}^{*}-d\right)_{+}\right] \quad \text { for all } d \in \mathbb{R},
$$

which proves (10).

Applying (10) inductively, we get $X_{i}^{*}+X_{j}^{*} \stackrel{d}{=} X_{i}^{M}+X_{j}^{M}$ for all $i \neq j$. By Theorem 4.1, $X_{i}^{M}+X_{j}^{M}$ is a counter-monotonic sum in $\mathcal{R}\left(F_{i}, F_{j}\right)$, so it follows from Lemma 2.3 that $\left(X_{i}^{*}, X_{j}^{*}\right)$ is counter-monotonic for all $i \neq j$. Using Theorem 4.1 once more, we conclude that $\mathbf{X}^{*}$ is a mutually exclusive (from below) random vector.

Theorem 5.1 can be regarded as a complement of Lemmas 2.2 (c) and 2.3. The optimality of the sum of a random vector's components in the sense of convex order, under appropriate conditions, allows one to identify the dependence structure of that random vector.

Corollary 5.2. Let $\mathbf{X}^{*}=\left(X_{1}^{*}, \ldots, X_{n}^{*}\right)$ be a fixed random vector in the Fréchet space $\mathcal{R}\left(F_{1}, \ldots, F_{n}\right)$ which satisfies Condition $(A)$ and $S^{*}=X_{1}^{*}+\cdots+X_{n}^{*}$. Then $S^{*} \stackrel{d}{=} S^{M}$ if and only if $\mathbf{X}^{*}$ is mutually exclusive.

If each marginal distribution has finite second moment, then the minimal convex sum property is equivalent to the minimality of the variance of the sum of a random vector's components.

Proposition 5.3. Let $\mathbf{X}^{*}=\left(X_{1}^{*}, \ldots, X_{n}^{*}\right)$ be a fixed random vector in the Fréchet space $\mathcal{R}\left(F_{1}, \ldots, F_{n}\right)$ of square integrable distributions such that Condition $(A)$ is fulfilled. Then $\mathbf{X}^{*}$ is mutually exclusive if and only if

$$
\operatorname{Var}\left(X_{1}^{*}+\cdots+X_{n}^{*}\right) \leq \operatorname{Var}\left(X_{1}+\cdots+X_{n}\right) \quad \text { for all }\left(X_{1}, \ldots, X_{n}\right) \in \mathcal{R}\left(F_{1}, \ldots, F_{n}\right) .
$$

Proof. The "only if" part is trivial. For the "if" part, assume that the minimum of $\operatorname{Var}\left(X_{1}+\right.$ $\left.\cdots+X_{n}\right)$ over all $\left(X_{1}, \ldots, X_{n}\right) \in \mathcal{R}\left(F_{1}, \ldots, F_{n}\right)$ is attained by $\mathbf{X}^{*}=\left(X_{1}^{*}, \ldots, X_{n}^{*}\right)$, but $\mathbf{X}^{*}$ is not mutually exclusive. By Theorem 4.1 , we can find some $\left(X_{i}^{*}, X_{j}^{*}\right)$ which is not countermonotonic, or equivalently, $\operatorname{Cov}\left(X_{i}^{*}, X_{j}^{*}\right)$ is not minimal. Considering the mutually exclusive random vector $\mathbf{X}^{M}=\left(X_{1}^{M}, \ldots, X_{n}^{M}\right)$, whose existence is guaranteed by Condition (A), we have

$$
\operatorname{Var}\left(X_{1}^{*}+\cdots+X_{n}^{*}\right)>\operatorname{Var}\left(X_{1}^{M}+\cdots+X_{n}^{M}\right),
$$

which is a contradiction.

As a matter of fact, Corollary 5.2 and Proposition 5.3 admit the following generalization, taking into account Theorem 3.A.60 of Shaked and Shanthikumar (2007).

Proposition 5.4. Let $\mathbf{X}^{*}=\left(X_{1}^{*}, \ldots, X_{n}^{*}\right)$ be a fixed random vector in the Fréchet space $\mathcal{R}\left(F_{1}, \ldots, F_{n}\right)$ such that Condition $(A)$ is fulfilled. Then $\mathbf{X}^{*}$ is mutually exclusive if and only if

$$
\mathbb{E}\left[\phi\left(X_{1}^{*}+\cdots+X_{n}^{*}\right)\right]=\mathbb{E}\left[\phi\left(X_{1}^{M}+\cdots+X_{n}^{M}\right)\right]
$$

for some strictly convex function $\phi$ such that the expectations exist. 
We caution that while the "only if" part of Theorem 5.1, Corollary 5.2, Propositions 5.3 and 5.4 is always true even without Condition (A), the imposition of Condition (A) is crucial for the reverse implication to hold. In general, the minimality of the sum of the components of a random vector with respect to convex order without knowing any properties of the underlying Fréchet space is not sufficient for mutual exclusivity, as Example 5.6 below illustrates.

Definition 5.5. (a) (Wang and Wang (2011), Definition 2.1) A distribution function $F$ is said to be $n$-completely mixable if there exist $n$ random variables $X_{1}, \ldots, X_{n}$ with the same distribution $F$ such that

$$
\mathbb{P}\left(X_{1}+\cdots+X_{n}=C\right)=1
$$

for some constant $C \in \mathbb{R}$.

(b) A Fréchet space $\mathcal{R}\left(F_{1}, \ldots, F_{n}\right)$ is said to be completely mixable if there exist $n$ random variables $X_{1}, \ldots, X_{n}$ with distribution functions $F_{1}, \ldots, F_{n}$ respectively such that (14) holds for some $C \in \mathbb{R}$.

Example 5.6. Consider the Fréchet space $\mathcal{R}(F, \ldots, F)$, where $F$ is the binomial distribution with parameters $m$ and $p / n$, and $m \in \mathbb{N} \backslash\{1\}$ and $p, n \in \mathbb{N}$. The non-degeneracy of $F$ requires that $p<n$, or $p+1 \leq n$. By Proposition 2.3 (3) of Wang and Wang (2011), $F$ is $n$-completely mixable, so there exists a random vector $\mathbf{X}^{*}=\left(X_{1}^{*}, \ldots, X_{n}^{*}\right) \in \mathcal{R}(F, \ldots, F)$ such that $X_{1}^{*}+\cdots+X_{n}^{*}$ is almost surely constant. By Jensen's inequality,

$$
X_{1}^{*}+\cdots+X_{n}^{*} \leq_{c x} X_{1}+\cdots+X_{n}
$$

for all $\left(X_{1}, \ldots, X_{n}\right) \in \mathcal{R}(F, \ldots, F)$. However, the inequalities

$$
\sum_{i=1}^{n} \mathbb{P}\left(X_{i}^{*}>0\right)=n\left[1-\left(1-\frac{p}{n}\right)^{m}\right]>n\left[1-\left(1-\frac{p}{n}\right)\right]=p \geq 1
$$

and

$$
\sum_{i=1}^{n} \mathbb{P}\left(X_{i}^{*}<m\right)=n\left[1-\left(\frac{p}{n}\right)^{m}\right]>n\left(1-\frac{p}{n}\right)=n-p \geq 1
$$

show that Condition (A) is violated. In other words, $\mathbf{X}^{*}$ is not a mutually exclusive random vector.

For further information about complete mixability, we refer the reader to Puccetti et al. (2012) and Wang et al. (2013).

From Theorem 5.1 and Example 5.6, one sees that mutual exclusivity and complete mixability are possible solutions to the convex minimization problem

$$
\min _{\left(X_{1}, \ldots, X_{n}\right) \in \mathcal{R}\left(F_{1}, \ldots, F_{n}\right)} \mathbb{E}\left[f\left(X_{1}+\cdots+X_{n}\right)\right]
$$

where $f$ is a given convex function, for different types of marginals $F_{1}, \ldots, F_{n}$. More precisely:

- if $\mathcal{R}\left(F_{1}, \ldots, F_{n}\right)$ satisfies Condition $(\mathrm{A})$, then mutual exclusivity will be the optimal dependence structure; 
- if $\mathcal{R}\left(F_{1}, \ldots, F_{n}\right)$ is completely mixable, the solution will be complete mixability.

Interestingly, mutual exclusivity and complete mixability are not "mutually exclusive" dependence structures. As we shall show in the next proposition, their coexistence is equivalent to the underlying marginal distributions being two-point distributions.

Proposition 5.7. A Fréchet space $\mathcal{R}\left(F_{1}, \ldots, F_{n}\right)$ is completely mixable and accommodates mutually exclusive from below random vectors if and only if

$$
F_{i}(x)=\left(1-q_{i}\right) 1_{\left\{x \geq l_{i}\right\}}+q_{i} 1_{\left\{x \geq u_{i}\right\}},
$$

where the essential infima $l_{1}, \ldots, l_{n}$ and essential suprema $u_{1}, \ldots, u_{n}$ satisfy $u_{1}-l_{1}=\cdots=$ $u_{n}-l_{n}$ and $\sum_{i=1}^{n} q_{i}=1$.

Proof. Suppose that the mutually exclusive from below random vector $\mathbf{X}^{M B}$ exists in the completely mixable Fréchet space $\mathcal{R}\left(F_{1}, \ldots, F_{n}\right)$. Then it follows from Corollary 5.2 that the mutually exclusive sum $S^{M B}$ is almost surely equal to its mean. Define, for $i=1, \ldots, n$, $A_{i}:=\left\{X_{i}^{M B}>l_{i}\right\}$. For any fixed $i \in\{1, \ldots, n\}$ and $\omega \in A_{i}$, mutual exclusivity implies that $X_{j}^{M B}(\omega)=l_{j}$ for all $j \neq i$. If $X_{i}^{M B}$ can take two or more distinct values on $A_{i}$, then this would contradict the degeneracy of $S^{M B}$. Hence, each $X_{i}^{M B}$ can only take two values, namely, $l_{i}$ and $u_{i}$. Now if $\omega_{i} \in A_{i}$ and $\omega_{j} \in A_{j}$ with $i \neq j$, then

$$
u_{i}-l_{i}+\sum_{i=1}^{n} l_{i}=S^{M B}\left(\omega_{i}\right)=S^{M B}\left(\omega_{j}\right)=u_{j}-l_{j}+\sum_{j=1}^{n} l_{j},
$$

showing that $u_{i}-l_{i}$ must equal the same positive constant, say $c$, for all $i=1, \ldots, n$. Since $\sum_{i=1}^{n} l_{i}+c=S^{M B}=\mathbb{E}\left[S^{M B}\right]=\sum_{i=1}^{n} l_{i}+c \sum_{i=1}^{n} q_{i}$, we have $\sum_{i=1}^{n} q_{i}=1$.

Conversely, if the distribution of each $F_{i}$ is given as in (15) with the stated properties, then Condition (A) is satisfied, guaranteeing the existence of the mutually exclusive from below random vector $\mathbf{X}^{M B}$ in $\mathcal{R}\left(F_{1}, \ldots, F_{n}\right)$. As $\sum_{i=1}^{n} q_{i}=1$, the sets $A_{i}$ defined in the "only if" part of the proof are mutually exclusive and exhaustive. We conclude that $S^{M B}=\sum_{i=1}^{n} l_{i}+c$ almost surely, where $c:=u_{1}-l_{1}=\cdots=u_{n}-l_{n}$. Hence $\mathcal{R}\left(F_{1}, \ldots, F_{n}\right)$ is completely mixable.

Using the same argument, it can be easily shown that a Fréchet space $\mathcal{R}\left(F_{1}, \ldots, F_{n}\right)$ is completely mixable and accommodates mutually exclusive from above random vectors if and only if

$$
F_{i}(x)=p_{i} 1_{\left\{x \geq l_{i}\right\}}+\left(1-p_{i}\right) 1_{\left\{x \geq u_{i}\right\}},
$$

such that $u_{1}-l_{1}=\cdots=u_{n}-l_{n}$ and $\sum_{i=1}^{n} p_{i}=1$.

From its definition, complete mixability is a negative dependence structure. It is also clear that when the underlying Fréchet space is completely mixable, the minimal convex sum property characterizes complete mixability. However, necessary and sufficient conditions for complete mixability are not known in the literature. In addition, a completely mixable random vector may not be pairwise counter-monotonic. 
Example 5.8. Consider $n=3$ and $\Omega=\left\{\omega_{1}, \omega_{2}\right\}$. Define a random vector $\mathbf{X}^{*}=\left(X_{1}^{*}, X_{2}^{*}, X_{3}^{*}\right)$ via:

\begin{tabular}{c|ccc|c|c}
\hline$\omega$ & $X_{1}^{*}(\omega)$ & $X_{2}^{*}(\omega)$ & $X_{3}^{*}(\omega)$ & $\left(X_{1}^{*}+X_{2}^{*}+X_{3}^{*}\right)(\omega)$ & $\mathbb{P}(\{\omega\})$ \\
\hline$\omega_{1}$ & 1 & 2 & -3 & 0 & $1 / 2$ \\
$\omega_{2}$ & -2 & -1 & 3 & 0 & $1 / 2$ \\
\hline
\end{tabular}

As $X_{1}^{*}+X_{2}^{*}+X_{3}^{*} \equiv 0, \mathbf{X}^{*}$ is completely mixable. However, $\left(X_{1}^{*}, X_{2}^{*}\right)$ is not counter-monotonic.

As we shall show in the next section, mutual exclusivity possesses a distributional representation that can be readily compared with that in Lemma $2.2(\mathrm{~d})$. Corresponding precisely to the Fréchet lower bound and enjoying a counterpart of each property in Lemma 2.2 as well as a high degree of analytic tractability, mutual exclusivity, whenever it exists, is arguably the strongest dependence structure.

\section{Distributional representation of a mutually exclusive random vector}

In this section, we present the third characterization of mutual exclusivity by demonstrating the distributional representation of mutually exclusive random variables. This result is of both practical and theoretical importance because it not only provides a simple method for generating mutually exclusive random variables, but also reveals the underlying copula which is unknown in the literature. As in the extreme cases of comonotonicity and counter-monotonicity, a single uniform $(0,1)$ random variable suffices to generate the whole mutually exclusive random vector.

Theorem 6.1. Suppose that the Fréchet space $\mathcal{R}\left(F_{1}, \ldots, F_{n}\right)$ satisfies Condition (A) and $U$ is any uniform $(0,1)$ random variable.

(a) (Mutual exclusivity from below) If $\sum_{i=1}^{n} q_{i} \leq 1$, then

$$
\mathbf{X}^{M B} \stackrel{d}{=}\left(F_{1}^{-1}\left(U_{1}\right), \ldots, F_{n}^{-1}\left(U_{n}\right)\right)
$$

where, for $i=1, \ldots, n$,

$$
U_{i}:=\left(U+1-\sum_{j=1}^{i} q_{j}\right) 1_{\left\{U \in\left(0, \sum_{j=1}^{i} q_{j}\right)\right\}}+(1-U) 1_{\left\{U \in\left[\sum_{j=1}^{i} q_{j}, 1\right)\right\}}
$$

Moreover,

$$
F_{i}^{-1}\left(U_{i}\right)= \begin{cases}F_{i}^{-1}\left(U+1-\sum_{j=1}^{i} q_{j}\right), & \sum_{j=1}^{i-1} q_{j} \leq U<\sum_{j=1}^{i} q_{j}, \\ l_{i}, & \text { otherwise, }\end{cases}
$$

with the convention that $\sum_{j=1}^{0} q_{j}:=0$; 
(b) (Mutual exclusivity from above) if $\sum_{i=1}^{n} p_{i} \leq 1$, then

$$
\mathbf{X}^{M A} \stackrel{d}{=}\left(F_{1}^{-1}\left(U_{1}\right), \ldots, F_{n}^{-1}\left(U_{n}\right)\right),
$$

where, for $i=1, \ldots, n$,

$$
U_{i}:=\left(\sum_{j=1}^{i} p_{j}-U\right) 1_{\left\{U \in\left(0, \sum_{j=1}^{i} p_{j}\right)\right\}}+U 1_{\left\{U \in\left[\sum_{j=1}^{i} p_{j}, 1\right)\right\}} .
$$

Furthermore,

$$
F_{i}^{-1}\left(U_{i}\right)= \begin{cases}F_{i}^{-1}\left(\sum_{j=1}^{i} p_{j}-U\right), & \sum_{j=1}^{i-1} p_{j} \leq U<\sum_{j=1}^{i} p_{j}, \\ u_{i}, & \text { otherwise }\end{cases}
$$

with the convention that $\sum_{j=1}^{0} p_{j}:=0$.

Proof. We only prove (a) as the proof of (b) is similar. We first check that the distribution of each $F_{i}^{-1}\left(U_{i}\right)$ is indeed $F_{i}$ by showing that each $U_{i}$ is a uniform $(0,1)$ random variable. To this end, we compute the characteristic function of $U_{i}$ :

$$
\begin{aligned}
\varphi_{U_{i}}(t) & =\int_{0}^{\sum_{j=1}^{i} q_{j}} e^{i t\left(p+1-\sum_{j=1}^{i} q_{j}\right)} \mathrm{d} p+\int_{\sum_{j=1}^{i} q_{j}}^{1} e^{i t(1-p)} \mathrm{d} p \\
& =\left(\frac{e^{i t}-e^{i t\left(1-\sum_{j=1}^{i} q_{j}\right)}}{i t}\right)+\left(\frac{e^{i t\left(1-\sum_{j=1}^{i} q_{j}\right)}-1}{i t}\right) \\
& =\frac{e^{i t}-1}{i t}, \quad t \neq 0 .
\end{aligned}
$$

Due to the one-to-one correspondence between characteristic functions and distributions, we deduce that each $U_{i}$ is a uniform $(0,1)$ random variable, and by extension, $\left(F_{1}^{-1}\left(U_{1}\right), \ldots, F_{n}^{-1}\left(U_{n}\right)\right)$ lies in $\mathcal{R}\left(F_{1}, \ldots, F_{n}\right)$.

Next, we prove $(17)$ by showing that $F_{i}^{-1}\left(U_{i}\right)=l_{i}$ whenever $U \notin\left[\sum_{j=1}^{i-1} q_{j}, \sum_{j=1}^{i} q_{j}\right)$ for each $i=1, \ldots, n$.

- If $0<U<\sum_{j=1}^{i-1} q_{j}$, then $1-\sum_{j=1}^{i} q_{j}<U+1-\sum_{j=1}^{i} q_{j}<1-q_{i}$, so $F_{i}^{-1}\left(U_{i}\right)=l_{i}$.

- If $\sum_{j=1}^{i} q_{j} \leq U<1$, then $0<1-U \leq 1-\sum_{j=1}^{i} q_{j} \leq 1-q_{i}$. Thus $F_{i}^{-1}\left(U_{i}\right)=l_{i}$ as well.

As the intervals $\left[0, q_{1}\right),\left[q_{1}, q_{1}+q_{2}\right), \ldots,\left[\sum_{i=1}^{n-1} q_{i}, \sum_{i=1}^{n} q_{i}\right)$ are disjoint, it follows from (17) that

$$
\mathbb{P}\left(F_{i}^{-1}\left(U_{i}\right)>l_{i}, F_{j}^{-1}\left(U_{j}\right)>l_{j}\right)=0 \text { for all } i \neq j .
$$

Consequently, $\left(F_{1}^{-1}\left(U_{1}\right), \ldots, F_{n}^{-1}\left(U_{n}\right)\right)$ is mutually exclusive from below, as desired.

We give several remarks to elucidate the seemingly complicated distributional representation in Theorem 6.1. 
Remark 6.2. (a) Note that $U_{1}, \ldots, U_{n}$ all depend on the same uniform $(0,1)$ random variable $U$, but they are not monotonic functions of $U$. As in the case of comonotonicity, a mutually exclusive random vector $\mathbf{X}^{M}$ admits a simple structure in the sense that calculations of expectations involving $\mathbf{X}^{M}$ are particularly easy since only a single integration is necessary (see the remarks following Theorem 7.1 in the next section).

(b) The distributional representation is not unique since it is possible to rearrange the sequence of probabilities $\left\{q_{1}, \ldots, q_{n}\right\}$ and $\left\{p_{1}, \ldots, p_{n}\right\}$ to produce different non-overlapping intervals on which the value of at most one random variable can exceed (resp. be less than) its essential infimum (resp. essential supremum).

(c) In the context of such a distributional representation, the meaning of Condition (A) manifests itself since the inequality $\sum_{i=1}^{n} q_{i} \leq 1$ or $\sum_{i=1}^{n} p_{i} \leq 1$ ensures that the randomization variable $U$ is bounded above by one.

(d) If the underlying probability space is atomless, then we can apply Corollary 6.11 of Kallenberg (2002) to strengthen the distributional representation in Theorem 6.1 into an almost sure equality: there exists a uniform $(0,1)$ random variable $V$ such that

$$
\mathbf{X}^{M B}=\left(F_{1}^{-1}\left(V_{1}\right), \ldots, F_{n}^{-1}\left(V_{n}\right)\right) \quad \text { almost surely }
$$

where, for $i=1, \ldots, n$,

$$
V_{i}:=\left(V+1-\sum_{j=1}^{i} q_{j}\right) 1_{\left\{V \in\left(0, \sum_{j=1}^{i} q_{j}\right)\right\}}+(1-V) 1_{\left\{V \in\left[\sum_{j=1}^{i} q_{j}, 1\right)\right\}} .
$$

(e) From the definition of $U_{1}, \ldots, U_{n}$, the copula of a mutually exclusive random vector, denoted by $C_{n}^{M}\left(\cdot, \ldots, \cdot \mid q_{1}, \ldots, q_{n}\right)$, can in principle be determined. In the case of mutual exclusivity from below, for instance, if $u_{i} \in\left[1-\wedge_{j=1}^{n} q_{j}, 1\right]$ for all $i=1, \ldots, n$, then

$$
\begin{aligned}
C_{n}^{M B}\left(u_{1}, \ldots, u_{n} \mid q_{1}, \ldots, q_{n}\right) & =\mathbb{P}\left(U_{1} \leq u_{1}, \ldots, U_{n} \leq u_{n}\right) \\
& =\mathbb{P}\left(U \in \bigcup_{i=1}^{n}\left[\sum_{j=1}^{i-1} q_{j}, u_{i}^{*}\right] \cup\left[\sum_{i=1}^{n} q_{i}, 1\right]\right)
\end{aligned}
$$

where $u_{i}^{*}:=u_{i}-1+\sum_{j=1}^{i} q_{j} \in\left[\sum_{j=1}^{i-1} q_{j}, \sum_{j=1}^{i} q_{j}\right)$ (see Figure 1 for an illustration when $n=3)$. It follows that

$$
\begin{aligned}
C_{n}^{M B}\left(u_{1}, \ldots, u_{n} \mid q_{1}, \ldots, q_{n}\right) & =\sum_{i=1}^{n}\left(u_{i}^{*}-\sum_{j=1}^{i-1} q_{j}\right)+\left(1-\sum_{i=1}^{n} q_{i}\right) \\
& =\sum_{i=1}^{n}\left(u_{i}-1+q_{i}\right)+\left(1-\sum_{i=1}^{n} q_{i}\right) \\
& =\sum_{i=1}^{n} u_{i}-(n-1) .
\end{aligned}
$$

The general expression of $C_{n}^{M B}\left(u_{1}, \ldots, u_{n} \mid q_{1}, \ldots, q_{n}\right)$ depends on the ranges of values of $u_{1}, \ldots, u_{n}$. 


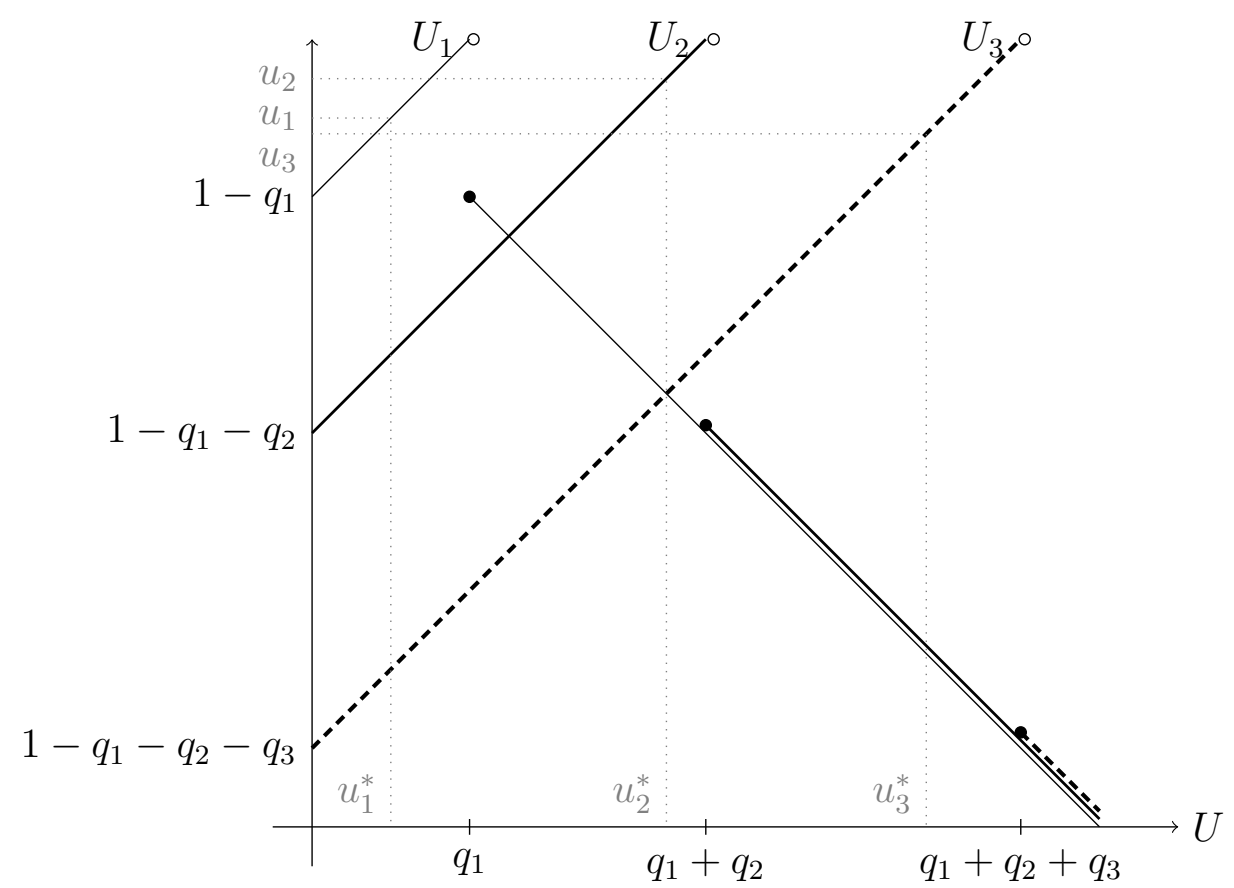

Figure 1: Illustration of the determination of $C_{3}^{M B}\left(u_{1}, \ldots, u_{3}\right)$ for $u_{i} \geq 1-\wedge_{j=1}^{3} q_{j}, i=1,2,3$.

Example 6.3. (Explicit calculations of $C_{2}^{M B}\left(\cdot, \cdot \mid q_{1}, q_{2}\right)$ ) In this example, we compute the bivariate mutually exclusive (from below) copula explicitly. Under the assumption that $q_{1} \leq q_{2}$ with $q_{1}+q_{2} \leq 1$, the expressions of $C_{2}^{M B}\left(u_{1}, u_{2} \mid q_{1}, q_{2}\right)$ for different $u_{1}$ and $u_{2}$ are given in Table 1.

For example, when $u_{1} \in\left[1-q_{1}-q_{2}, 1-q_{1}\right)$ and $u_{2} \in\left[1-q_{1}, 1\right]$, then

$$
\begin{aligned}
C_{2}^{M B}\left(u_{1}, u_{2} \mid q_{1}, q_{2}\right) & =\mathbb{P}\left(1-u_{1} \leq U \leq u_{2}+q_{1}+q_{2}-1\right)+\mathbb{P}\left(q_{1}+q_{2} \leq U \leq 1\right) \\
& =\left(u_{1}+u_{2}+q_{1}+q_{2}-2\right)_{+}+\left(1-q_{1}-q_{2}\right) \\
& =\left(u_{1}+u_{2}-1\right) \vee\left(1-q_{1}-q_{2}\right) .
\end{aligned}
$$

The calculations in other cases are similar.

\begin{tabular}{l|ccc}
\hline$C_{2}^{M B}\left(u_{1}, u_{2} \mid q_{1}, q_{2}\right)$ & $u_{2} \in\left[1-q_{1}, 1\right]$ & $u_{2} \in\left[1-q_{1}-q_{2}, 1-q_{1}\right)$ & $u_{2} \in\left[0,1-q_{1}-q_{2}\right)$ \\
\hline$u_{1} \in\left[1-q_{1}, 1\right]$ & $u_{1}+u_{2}-1$ & $\left(u_{1}-q_{2}\right) \wedge u_{2}+\left(u_{2}+q_{2}-1\right)_{+}$ & $u_{2}$ \\
$u_{1} \in\left[1-q_{1}-q_{2}, 1-q_{1}\right)$ & $\left(u_{1}+u_{2}-1\right) \vee\left(1-q_{1}-q_{2}\right)$ & $\left(u_{1}+u_{2}-1\right) \vee\left(1-q_{1}-q_{2}\right)$ & $u_{2}$ \\
$u_{1} \in\left[0,1-q_{1}-q_{2}\right)$ & $u_{1}$ & $u_{1}$ & $u_{1} \wedge u_{2}$ \\
\hline
\end{tabular}

Table 1: Explicit expressions of $C_{2}^{M B}\left(u_{1}, u_{2} \mid q_{1}, q_{2}\right)$ for different ranges of values of $u_{1}$ and $u_{2}$.

Note that $C_{2}^{M B}\left(\cdot, \cdot \mid q_{1}, q_{2}\right)$ is different from the Fréchet lower copula defined by $C_{M}\left(u_{1}, u_{2}\right):=$ $\left(u_{1}+u_{2}-1\right)_{+}$. In other words, there are two different copulas, $C_{2}^{M B}\left(\cdot, \cdot \mid q_{1}, q_{2}\right)$ and $C_{M}$, 
corresponding to the Fréchet lower bound $M_{2}$ :

$$
M_{2}\left(x_{1}, x_{2}\right)=C_{2}^{M B}\left(F_{1}\left(x_{1}\right), F_{2}\left(x_{2}\right) \mid q_{1}, q_{2}\right)=C_{M}\left(F_{1}\left(x_{1}\right), F_{2}\left(x_{2}\right)\right)
$$

for all $x_{1}, x_{2} \in \mathbb{R}$. No contradiction with Sklar's theorem (see, for example, Theorem 2.3.3 of Nelsen (2006)) arises; this example just presents another instance of the non-uniqueness of copulas corresponding to a given joint distribution if its marginals are not continuous. However, when $u_{i} \geq 1-q_{1} \geq 1-q_{2}$ for $i=1,2, C_{2}^{M B}\left(u_{1}, u_{2} \mid q_{1}, q_{2}\right)=C_{M}\left(u_{1}, u_{2}\right)$, showing that the two copulas agree on the product of the ranges of the marginal distribution functions. Note that unlike $C_{M}$, the copula $C_{2}^{M B}\left(\cdot, \cdot \mid q_{1}, q_{2}\right)$ is marginal-dependent in the sense that its definition involves the probabilities $q_{1}$ and $q_{2}$ from the marginal distributions.

\section{Characteristic function of a mutually exclusive sum}

Unlike comonotonicity, the characteristic function, and more generally, any transform functions whenever they exist, of a mutually exclusive sum will be shown in this section to comprise the sum of the marginal characteristic functions, as opposed to a product in the well-known case of independence. Of equal importance is that the consideration of characteristic functions allows us to give an equivalent reformulation of Condition (A) in terms of marginal characteristic functions. For simplicity, we consider mutual exclusivity from below with all essential infima being zero and from above with zero essential suprema. The general case can be treated by simple translation of random variables.

Theorem 7.1. Suppose that $\mathcal{R}\left(F_{1}, \ldots, F_{n}\right)$ is a Fréchet space with $l_{1}=\cdots=l_{n}=0$ or $u_{1}=\cdots=u_{n}=0$. Then $\mathcal{R}\left(F_{1}, \ldots, F_{n}\right)$ supports mutual exclusivity if and only if

$$
\sum_{i=1}^{n} \varphi_{i}(t)-(n-1), \quad t \in \mathbb{C},
$$

defines a valid characteristic function.

Proof. We consider the cases (1) $l_{1}=\cdots=l_{n}=0$ and (2) $u_{1}=\cdots=u_{n}=0$ separately.

Case 1. Assume that mutual exclusivity from below is admissible in $\mathcal{R}\left(F_{1}, \ldots, F_{n}\right)$. The characteristic function of the mutually exclusive sum $S^{M B}$ is

$$
\begin{aligned}
\varphi_{S^{M B}}(t) & =\mathbb{E}\left[e^{i t S^{M B}}\right] \\
& =\sum_{i=1}^{n} \mathbb{E}\left[e^{i t S^{M B}} \mid X_{i}>0\right] \mathbb{P}\left(X_{i}>0\right)+\mathbb{P}(S=0) \\
& =\sum_{i=1}^{n} \mathbb{E}\left[e^{i t X_{i}} \mid X_{i}>0\right] \mathbb{P}\left(X_{i}>0\right)+\mathbb{P}(S=0) \\
& =\sum_{i=1}^{n}\left[\varphi_{i}(t)-\mathbb{P}\left(X_{i}=0\right)\right]+\mathbb{P}(S=0)
\end{aligned}
$$


As $\mathbb{P}(S=0)=1-\mathbb{P}(S>0)=1-\sum_{i=1}^{n} \mathbb{P}\left(X_{i}>0\right)=1-\sum_{i=1}^{n}\left[1-\mathbb{P}\left(X_{i}=0\right)\right]$, we further have

$$
\begin{aligned}
\varphi_{S^{M B}}(t) & =\sum_{i=1}^{n}\left[\varphi_{i}(t)-\mathbb{P}\left(X_{i}=0\right)\right]+\left[1-\sum_{i=1}^{n}\left[1-\mathbb{P}\left(X_{i}=0\right)\right]\right] \\
& =\sum_{i=1}^{n} \varphi_{i}(t)-(n-1) .
\end{aligned}
$$

Conversely, suppose that $\sum_{i=1}^{n} \varphi_{i}-(n-1)$ is a valid characteristic function of a random variable, say $Y$. For any non-negative $\lambda$, we set $t=i \lambda$ to obtain

$$
\mathbb{E}\left[e^{-\lambda Y}\right]=\sum_{i=1}^{n} \mathbb{E}\left[e^{-\lambda X_{i}}\right]-(n-1)
$$

for all $\left(X_{1}, \ldots, X_{n}\right) \in \mathcal{R}\left(F_{1}, \ldots, F_{n}\right)$. Note that

$$
\begin{aligned}
\mathbb{E}\left[e^{-\lambda X_{i}}\right] & =\mathbb{P}\left(X_{i}=0\right)+\mathbb{E}\left[e^{-\lambda X_{i}} \mid X_{i}>0\right] \mathbb{P}\left(X_{i}>0\right) \\
& \rightarrow \mathbb{P}\left(X_{i}=0\right)
\end{aligned}
$$

as $\lambda \rightarrow \infty$ by dominated convergence theorem. It follows from (18) that

$$
\sum_{i=1}^{n} \mathbb{P}\left(X_{i}=0\right)-(n-1)=\sum_{i=1}^{n}\left(1-q_{i}\right)-(n-1) \geq 0,
$$

or $\sum_{i=1}^{n} q_{i} \leq 1$, which is Condition (A).

Case 2. If $\mathcal{R}\left(F_{1}, \ldots, F_{n}\right)$ admits mutual exclusivity from above and $X_{1}^{M A}, \ldots, X_{n}^{M A}$ are random variables which are mutually exclusive from above with $u_{1}=\cdots=u_{n}=0$, then $-X_{1}^{M A}, \ldots,-X_{n}^{M A}$ are random variables that are mutually exclusive from below with zero essential infima. In this case, the characteristic function of the mutually exclusive sum $S^{M A}$ is

$$
\begin{aligned}
\varphi_{S^{M A}}(t) & =\varphi_{-S^{M A}}(-t) \\
& =\sum_{i=1}^{n} \varphi_{-X_{i}}(-t)-(n-1) \\
& =\sum_{i=1}^{n} \varphi_{X_{i}}(t)-(n-1),
\end{aligned}
$$

where the second equality follows from the "only if" part of Case 1.

To show that if $\sum_{i=1}^{n} \varphi_{i}-(n-1)$ is a valid characteristic function, then Condition (A) is fulfilled, we can apply the same argument in the "if" part of Case 1 with $t=-i \lambda$ for any non-negative $\lambda$.

In the proof of the "only if" part of Theorem 7.1, the characteristic function of the mutually exclusive sum can also be derived by means of the distributional representation in Theorem 
6.1. For example,

$$
\begin{aligned}
\varphi_{S^{M B}}(t)= & \int_{0}^{q_{1}} e^{i t\left[F_{1}^{-1}\left(p+1-q_{1}\right)\right]} \mathrm{d} p+\int_{q_{1}}^{q_{1}+q_{2}} e^{i t\left[F_{2}^{-1}\left(p+1-q_{1}-q_{2}\right)\right]} \mathrm{d} p \\
& +\cdots+\int_{q_{1}+\cdots+q_{n-1}}^{q_{1}+\cdots+q_{n}} e^{i t\left[F_{n}^{-1}\left(p+1-q_{1}-\cdots-q_{n}\right)\right]} \mathrm{d} p \\
& \quad+\left[1-\left(q_{1}+\cdots+q_{n}\right)\right] \\
= & \sum_{i=1}^{n} \int_{\sum_{j=1}^{i-1} q_{j}}^{\sum_{j=1}^{i} q_{j}}\left(e^{i t\left[F_{i}^{-1}\left(p+1-\sum_{j=1}^{i} q_{j}\right)\right]}-1\right) \mathrm{d} p+1 \\
= & \sum_{i=1}^{n}\left(\varphi_{i}(t)-1\right)+1 \\
= & \sum_{i=1}^{n} \varphi_{i}(t)-(n-1) .
\end{aligned}
$$

In essence, this is merely the conditioning argument in the proof of Theorem 7.1 expressed equivalently in integral forms.

Armed with Theorem 7.1 and our preceding characterization of mutual exclusivity by the minimal convex sum property, we can also characterize mutually exclusive random vectors by the characteristic function of the sum of their components. Note that Condition (A) is not imposed a priori.

Theorem 7.2. A random vector $\mathbf{X}^{*}=\left(X_{1}^{*}, \ldots, X_{n}^{*}\right)$ in a Fréchet space $\mathcal{R}\left(F_{1}, \ldots, F_{n}\right)$ with $l_{1}=\cdots=l_{n}=0$ or $u_{1}=\cdots=u_{n}=0$ is mutually exclusive if and only if the characteristic function of $S^{*}=X_{1}^{*}+\cdots+X_{n}^{*}$ is given by

$$
\varphi_{S^{*}}(t)=\sum_{i=1}^{n} \varphi_{i}(t)-(n-1)
$$

for all $t \in \mathbb{C}$.

Proof. The necessity has been shown in the proof of Theorem 7.1. To prove the sufficiency, we assume that the characteristic function of $S^{*}$ is given as in (19). Then Theorem 7.1 implies that mutual exclusivity is supported by $\mathcal{R}\left(F_{1}, \ldots, F_{n}\right)$, or equivalently, Condition (A) is satisfied, guaranteeing the existence of a mutually exclusive random vector $\mathbf{X}^{M}$ in $\mathcal{R}\left(F_{1}, \ldots, F_{n}\right)$. By hypothesis and the necessity, $S^{*}$ coincides in distribution with $S^{M}$, so we conclude by Corollary 5.2 that $S^{*}$ must be mutually exclusive.

We end this section by applying Theorem 7.2 to show that a mutually exclusive sum of mixture distributions remains as a mixture distribution. The same holds for compound distributions. These results bear particular importance in risk theory.

Example 7.3. (Mutually exclusive sums of mixture distributions) Consider the random variables $X_{1}, \ldots, X_{n}$ distributed as

$$
X_{i} \stackrel{d}{=} \begin{cases}0, & \text { with probability } 1-q_{i}, \\ Y_{i}, & \text { with probability } q_{i},\end{cases}
$$


where $Y_{1}, \ldots, Y_{n}$ are strictly positive random variables. Such random variables $X_{1}, \ldots, X_{n}$ arise, for example, in the individual risk model in risk theory (see Chapter 2 of Kaas et al. (2008) for further discussions). Assume that $\sum_{i=1}^{n} q_{i} \leq 1$ so that Condition (A) is satisfied for the Fréchet space $\left(F_{X_{1}}, \ldots, F_{X_{n}}\right)$. By Theorem 7.2 , the characteristic function of the mutually exclusive sum $S^{M}=X_{1}^{M}+\cdots+X_{n}^{M}$ can be computed as

$$
\begin{aligned}
\varphi_{S^{M}}(t) & =\sum_{i=1}^{n} \varphi_{X_{i}}(t)-(n-1) \\
& =\sum_{i=1}^{n}\left[\left(1-q_{i}\right)+q_{i} \varphi_{Y_{i}}(t)\right]-(n-1) \\
& =\left[1-\sum_{i=1}^{n} q_{i}\right]+\sum_{i=1}^{n} q_{i} \varphi_{Y_{i}}(t),
\end{aligned}
$$

from which we deduce that $S^{M}$ is also a mixture random variable distributed as

$$
S^{M} \stackrel{d}{=} \begin{cases}0, & \text { with probability } 1-\sum_{i=1}^{n} q_{i}, \\ Y_{1}, & \text { with probability } q_{1}, \\ \vdots & \vdots \\ Y_{n}, & \text { with probability } q_{n} .\end{cases}
$$

Example 7.4. (Mutually exclusive sums of compound distributions) In the collective risk model (see Chapter 3 of Kaas et al. (2008) for further discussions), we consider the compound random variables $S_{1}, \ldots, S_{n}$ distributed as $S_{i} \stackrel{d}{=} \sum_{j=1}^{N_{i}} X_{i j}$, where $N_{1}, \ldots, N_{n}$ are counting random variables and $\left\{X_{i j}\right\}_{i, j \in \mathbb{N}}$ is a sequence of identically distributed non-negative random variables with characteristic function $\varphi_{X}$, such that $N_{i}$ and $\left\{X_{i j}\right\}_{j \geq 1}$ are independent for any given $i \in\{1, \ldots, n\}$. The characteristic function of each $S_{i}$ is given by

$$
\varphi_{S_{i}}(t)=P_{N_{i}}\left[\varphi_{X}(t)\right]
$$

where $P_{N_{i}}$ is the probability generating function of $N_{i}$. If $\left(F_{N_{1}}, \ldots, F_{N_{n}}\right)$ supports a mutually exclusive random vector, then so does $\left(F_{S_{1}}, \ldots, F_{S_{n}}\right)$ by virtue of Theorem 7.1. Then it follows from Theorem 7.2 that the characteristic function of the mutually exclusive sum $S^{M}=S_{1}^{M}+$ $\cdots+S_{n}^{M}$ of the compound variables is given by

$$
\varphi_{S^{M}}(t)=\sum_{i=1}^{n} P_{N_{i}}\left[\varphi_{X}(t)\right]-(n-1)=P_{N^{M}}\left[\varphi_{X}(t)\right],
$$

where $N^{M}:=N_{1}^{M}+\cdots+N_{n}^{M}$. In other words, $S^{M}$ is also a compound random variable, whose primary distribution is that of the mutually exclusive sum $N^{M}$ of the counting random variables, and the secondary distribution remains unchanged at $X$.

We remark that while the result in Example 7.3 can also be established by considering distribution functions and using a simple conditioning argument similar to that in the proof of Theorem 7.1 without resort to characteristic functions, such an approach may not work in Example 7.4. This is because the distribution function of a compound variable in terms of the probability functions of the primary and secondary distributions via convolution in general takes a complicated form. 


\section{Concluding remarks}

In this article, the investigation of mutual exclusivity initiated in Dhaene and Denuit (1999) is considerably expanded and several novel characterizations of mutually exclusive random variables are given. It has been shown that in a multi-dimensional setting, mutual exclusivity is the strongest negative dependence structure corresponding to the Fréchet lower bound and enjoying parallel defining properties of comonotonicity, under some conditions on the marginal distributions (Condition (A)).

It is well-known that risk measures and comonotonicity are intimately linked. A promising future research direction will be exploring the relationship between mutual exclusivity and risk measures such as Value-at-Risk and Tail Value-at-Risk, and the explicit expressions of these risk measures of mutually exclusive sums. The derivation of lower bounds on the Tail Value-at-Risk and Haezendonck-Goovaerts risk measures of a sum of random variables characterizing mutual exclusivity is pursued in Cheung and Lo (2013b).

\section{Acknowledgements}

The authors wish to thank the anonymous referee for careful reading and several valuable comments and suggestions which significantly improved the manuscript. Ka Chun Cheung was partially supported by a grant from the Research Grants Council of the Hong Kong Special Administrative Region, China (Project No. HKU 701213).

\section{References}

Cheung, K.C., 2008. Characterization of comonotonicity using convex order. Insurance: Mathematics and Economics 43, 403-406.

Cheung, K.C., 2010. Characterizing a comonotonic random vector by the distribution of the sum of its components. Insurance: Mathematics and Economics 47, 130-136.

Cheung, K.C., Dhaene, J., Lo, A., Tang, Q., 2014. Reducing risk by merging counter-monotonic risks. Insurance: Mathematics and Economics 54, 58-65.

Cheung, K.C., Lo, A., 2013a. Characterizations of counter-monotonicity and upper comonotonicity by (tail) convex order. Insurance: Mathematics and Economics 53, 334-342.

Cheung, K.C., Lo, A., 2013b. General lower bounds on convex functionals of aggregate sums. Insurance: Mathematics and Economics 53, 884-896.

Deelstra, G., Dhaene, J., Vanmaele, M., 2011. An overview of comonotonicity and its applications in finance and insurance, in: Nunno, G.D., Oksendal, B. (Eds.), Advanced Mathematical Methods for Finance. Springer, Germany, Heidelberg. 
Denuit, M., Dhaene, J., Goovaerts, M., Kaas, R., 2005. Actuarial Theory for Dependent Risks: Measures, Orders and Models. John Wiley \& Sons, Inc.

Dhaene, J., Denuit, M., 1999. The safest dependence structure among risks. Insurance: Mathematics and Economics 25, 11-21.

Dhaene, J., Denuit, M., Goovaerts, M., Kaas, R., Vyncke, D., 2002. The concept of comonotonicity in actuarial science and finance: theory. Insurance: Mathematics and Economics 31, $\underline{3-33 .}$

Dhaene, J., Goovaerts, M., 1996. Dependency of risks and stop-loss order. ASTIN Bulletin 26, 201-212.

Joe, H., 1997. Multivariate Models and Dependence Concepts. Chapman and Hall, London.

Kaas, R., Goovaerts, M., Dhaene, J., Denuit, M., 2008. Modern Actuarial Risk Theory Using R. Springer-Verlag, Berlin, Heidelberg.

Kallenberg, O., 2002. Foundations of Modern Probability. Springer-Verlag, New York.

Nelsen, R.B., 2006. An Introduction to Copulas. Springer, New York.

Puccetti, G., Wang, B., Wang, R., 2012. Advances in complete mixability. Journal of Applied Probability 49, 430-440.

Shaked, M., Shanthikumar, J.G., 2007. Stochastic Orders. Springer, New York.

Wang, B., Wang, R., 2011. The complete mixability and convex minimization problems with monotone marginal densities. Journal of Multivariate Analysis 102, 1344-1360.

Wang, R., Peng, L., Yang, J., 2013. Bounds for the sum of dependent risks and worst valueat-risk with monotone marginal densities. Finance and Stochastics 17, 395-417. 